\title{
Un contrôle de la qualité proche de la pratique
}

\section{Luise Menzi}

Dr phil., responsable réadaptation, ANQ, Association nationale pour le développement de la qualité dans les hôpitaux et les cliniques

Bien que les mesures de la qualité dans les hôpitaux et les cliniques soient imposées par la loi, elles ne font pas l'unanimité. Si, de prime abord, elles immobilisent des ressources, elles sont payantes à long terme. Des relevés de données judicieux et des comparaisons justes entre établissements exigent du temps et de l'expertise. L'ANQ œuvre dans ce sens depuis six ans, et les premiers résultats sont visibles. L'exemple de la réadaptation illustrera le chemin suivi.

L'ANQ réalise dans l'ensemble de la Suisse, sur mandat des partenaires tarifaires, des mesures uniformes dans le secteur résidentiel de trois domaines: médecine somatique aiguë, réadaptation et psychiatrie. Elle effectue des évaluations comparatives nationales et publie les résultats des mesures d'une manière transparente, dès que les données sont d'une qualité suffisante pour permettre des comparaisons justes des établissements. Le travail de l'ANQ vise à promouvoir des processus d'amélioration continus et à en lancer de nouveaux quand la nécessité s'en fait sentir.

\section{Une initiative du système de santé}

L'activité de l'ANQ se fonde sur la loi sur l'assurancemaladie (LAMal) de 1994. Celle-ci impose aux hôpitaux, aux cliniques, aux assureurs et aux cantons une assurance qualité définie de manière contractuelle, en les obligeant à contrôler régulièrement l'efficacité, l'adéquation et le caractère économique des prestations fournies. Ce contrôle peut notamment prendre la forme de comparaisons des hôpitaux et des cliniques, en particulier en ce qui concerne les coûts et la qualité médicale des résultats. En pratique, la manière de fournir la preuve de la qualité a été laissée au choix des fournisseurs de prestations et des agents payeurs. A cet effet, $\mathrm{H}+$ (l'association des hôpitaux) et santésuisse (celle des assureurs-maladie), les cantons et la principauté du Liechtenstein, la Conférence des directrices et directeurs cantonaux de la santé et les assurances sociales fédérales (assurance-accidents, assurance militaire et assurance-invalidité) ont fondé en 2009 une nouvelle association, l'ANQ. L'Office fédéral de la santé publique (OFSP) y siège en tant qu'observateur.
L'ANQ n'impose donc pas ses mesures de la qualité aux hôpitaux et aux cliniques, puisque ceux-ci en sont les initiateurs. Ses membres, ou plus exactement ses spécialistes, mettent au point et organisent l'ensemble des mesures et des processus, en s'efforçant de rester le plus près possible de la pratique: en réadaptation, par exemple, les mesures sont directement intégrées à la thérapie. Le but est de générer des données et de fixer des objectifs qui soient directement utilisables. Les relevés se font parallèlement au processus thérapeutique, ce qui permet d'exploiter concrètement les résultats des mesures, au lieu de se contenter de comparaisons théoriques suivies d'un archivage.

\section{Des thèmes de mesure reconnus dans l'ensemble du pays}

Les thèmes retenus pour chaque domaine sont définis dans le plan de mesures de l'ANQ. Ce plan, fruit d'une large collaboration avec la branche, est reconnu sur le plan national. Celui en usage en réadaptation repose sur l'expérience et les résultats des projets pilotes menés en réadaptation musculo-squelettique et neurologique (de 2006 à 2011), projets qui ont été élargis à l'ensemble de la Suisse en 2013. Depuis, pratiquement toutes les cliniques de réadaptation suisses participent aux mesures de l'ANQ. Un comité d'experts permanent, le groupe qualité réadaptation, suit et développe en continu le plan de mesures, en tenant compte des commentaires des cliniques.

\section{Une démarche axée sur les patients}

L'individualité des patients est au premier plan pour les enquêtes sur la qualité dans tous les domaines de la 
réadaptation, mais les instruments de mesure utilisés diffèrent.

En réadaptation musculo-squelettique et en réadaptation neurologique, les évaluations sont effectuées à l'entrée et à la sortie afin de mettre en évidence l'amélioration des capacités fonctionnelles des patients. L'enquête comporte une auto-évaluation des personnes à traiter et une évaluation par l'équipe de réadaptation. Les objectifs sont fixés en commun avec les patients, et leur atteinte fait l'objet d'une évaluation conjointe, inspirée de la classification de l'OMS qui sert de référence dans ce domaine, la CIF (classification internationale du fonctionnement).

Si tout se déroule comme prévu, le premier rapport comparatif national relatif à la réadaptation sera publié pendant l'été 2016.

Cette manière de procéder est habituelle aujourd'hui en réadaptation. Elle consiste à impliquer le plus possible les patients dans le programme thérapeutique et à leur laisser ainsi leur part de responsabilité dans la guérison. En pratique, cela signifie: au départ, l'équipe soignante définit avec eux l'objectif qu'il est possible d'atteindre pendant l'hospitalisation, par exemple retourner vivre à domicile de manière autonome, reprendre une activité professionnelle à temps plein ou à temps partiel, etc. Cet objectif de participation et son atteinte font l'objet d'une question dans les mesures de l'ANQ, parce qu'il constitue le fil rouge de la thérapie. On peut donc ensuite, lors d'une mesure ultérieure, poser des questions telles que «arrivez-vous à ouvrir une brique de lait?». Des questions de ce genre, qui peuvent paraître déplacées au premier abord, sont essentielles pour déterminer si les patients sont capables de maîtriser le quotidien.

En réadaptation cardiologique et en réadaptation pneumologique, on évalue les limitations fonctionnelles et leur évolution au cours de la thérapie au moyen d'instruments appropriés, tout en interrogeant les patients sur la perception qu'ils en ont.

\section{Un relevé des données permettant des comparaisons justes des hôpitaux et des cliniques}

Le CIRS (cumulative illness rating scale) est une échelle destinée à l'évaluation cumulée des maladies qui permet de recueillir d'autres données sur les patients dans tous les secteurs de la réadaptation. Chaque clinique transmet également à l'ANQ les données de l'OFS qui figurent dans la statistique des hôpitaux. En effet, pour pouvoir comparer d'une manière juste les résultats des hôpitaux et des cliniques, il faut tenir compte des risques. Pour cela, on élimine les facteurs qui influencent négativement ou positivement les résultats, mais sur lesquels les établissements ne peuvent pas agir. Exemple: la clientèle d'une clinique présente, à l'entrée, des handicaps plus importants ou des pathologies plus nombreuses que la clientèle d'une autre clinique. Sans ajustement aux risques, la première aurait de plus mauvais résultats que la seconde, même si ses résultats thérapeutiques sont meilleurs.

\section{De premiers résultats palpables}

En médecine somatique aiguë, l'ANQ publie des résultats de mesure pseudonymisés depuis 2012 et des résultats individuels par clinique depuis 2014, par exemple les taux d'infections du site opératoire après certaines interventions chirurgicales. En psychiatrie, les résultats ont été publiés sous forme résumée depuis 2013 et l'importance des symptômes en psychiatrie adultes devrait être présentée par clinique à partir de l'automne 2015. La publication de la qualité des résultats permet des comparaisons transparentes au niveau national. Les hôpitaux et les cliniques peuvent se fonder sur ces résultats pour prendre des mesures ciblées et ainsi améliorer leur qualité.

\section{Une phase de développement terminée}

Dans le dernier domaine où a elle a introduit des mesures, la réadaptation, l'ANQ a accompli un gros travail ces deux dernières années: tandis que les cliniques réalisaient les mesures, elle contrôlait en permanence et améliorait progressivement la qualité des données livrées, qui est la condition sine qua non d'une évaluation sérieuse. La phase d'introduction n'a pas encore fourni de résultats visibles, ce qui a été critiqué, mais il est normal que l'introduction d'un système de mesures uniforme prenne un certain temps. Ce temps était nécessaire et précieux, car il a permis de préparer soigneusement, avec les cliniques, un relevé et une documentation proches de la pratique.

Ce travail a porté ses fruits: cet été, l'ANQ a constaté que la qualité des données dans les cliniques de réadaptation était suffisante pour une première évaluation comparative nationale des résultats 2014 . Le groupe qualité est en train de définir les catégories d'analyse pour cette évaluation. Si tout se déroule comme prévu, le premier rapport comparatif national relatif à la réadaptation sera publié pendant l'été 2016. D'ici là, les mesures de l'ANQ auront pris leur place dans le processus thérapeutique de toutes les cliniques.

Disclosure statement

Membres de l'ANQ: H+ Les hôpitaux de Suisse; santésuisse; assurances sociales fédérales; cantons et principauté du Liechtenstein; Conférence des directrices et directeurs cantonaux de la santé 